\title{
Caracterización del regadío en la zona regable del Valle de Lemos (Lugo, España)
}

Recibido: 10 Abril 2007 / Aceptado: 21 Setembro 2007

(C) IBADER- Universidade de Santiago de Compostela 2007

Resumen El primer paso en la aplicación del Plan nacional de Regadíos (PNR) debe ser el análisis del uso del agua en los regadíos tradicionales. Para ello nos interesa definir las prácticas de riego habituales y evaluar la incidencia social del regadío en el ámbito rural. En este trabajo se presentan los resultados obtenidos en el estudio realizado en la zona regable del Valle de Lemos (Lugo, España). Se ha realizado una encuesta de campo incluyendo aspectos técnicos y sociales, así como evaluaciones de riego en parcelas de ensayo repartidas entre las clases de suelos existentes. Esta zona se caracteriza por una baja eficiencia en el uso del agua, unas pérdidas importantes en la red de distribución, una estructura atomizada de la propiedad y un escaso aprovechamiento de las infraestructuras existentes. A pesar de estos inconvenientes, la existencia de una importante red de distribución y la disponibilidad de agua hacen de la modernización de este regadío tradicional un reto que debemos afrontar.

Palabras clave Gestión del riego; Modernización; Parámetros sociales.

Abstract The first step towards the implementation of performance of the actions envisaged in the National Irrigation Plan (NIP) is to analyse water use in traditional irrigation. Moreover, the social impacts of irrigation on rural areas must be evaluated, and the common irrigation practices must be determined. This paper presents the results of a study conducted in the Lemos Valley irrigation district (Lugo, Spain). Irrigation evaluations were conducted

Tomas S. Cuesta $\cdot$ Xan X. Neira $\cdot$ Carlos J. Álvarez $\cdot$ Javier José Cancela

Departamento de Enxeñaría Agroforestal, Escola Politécnica Superior, Universidade de Santiago de Compostela. Campus Universitario s/n. E-27002 Lugo, España.

Tfno: +34-982-223996 ext 23290. Fax: +34-982-285926

e-mail: tcuesta@usc.es in nine trial sites, representing the existing soil types. A sample of irrigation users were interviewed to gather information about water use, land tenure and irrigation socioeconomics. This irrigation district is characterised by low water use efficiency, significant losses in the distribution network, fragmented land ownership and a poor use of the available infrastructure. Yet, water availability and an important distribution network render the modernisation of this traditional irrigated land a challenging task that must be faced.

Keywords Irrigation management; Modernisation; Social parameters.

\section{Introduction}

La demanda de agua en España, al igual que en todo el mundo, se ha ido incrementando en los últimos años, siendo la agricultura el sector que mayores necesidades presenta (Del Moral et al. 2003). Para lograr el mejor aprovechamiento de los recursos hidráulicos tradicionalmente se ha operado sobre la oferta de agua mediante la construcción de grandes obras de regulación y transporte de aguas superficiales (Vlachos, 2003). Algunos autores como Loucks (2000) resaltan que las nuevas corrientes en la gestión de los recursos hídricos van dirigidas a combinar las actuaciones sobre la oferta y las actuaciones sobre la demanda, reduciendo la cantidad de agua precisa para satisfacer esta última. Arrojo (2003) indica que estos hechos hacen pensar en la necesidad de destinar importantes recursos a la modernización de los regadíos tradicionales, entre los que se encuentran la mayor parte de los sistemas de riego por superficie.

Actualmente el regadío constituye un elemento fundamental de la estructuración del paisaje y es una de las variables territoriales que configuran decisivamente la demanda total de recursos hídricos (MIMAM, 1998). También destaca su importante función en las estrategias de desarrollo rural (Ortega et al., 2000) considerándose el agua como un activo ecosocial (Aguilera, 1994) que trasciende la concepción 
económica del riego hacia otras formulaciones de mayor utilidad social y sostenibilidad futura (Martín de Santa Olalla et al., 1999).

Bajo estas consideraciones, el Plan Nacional de Regadíos, PNR, establece actuaciones de modernización de los regadíos existentes, hasta el año 2008 , sobre una superficie que supera el millón de ha, con una inversión prevista de 6.113 millones de euros (MAPA, 2001). Según Beceiro (2003), con esta intervención se prevé que se produzca un ahorro de agua del orden de $2.700 \mathrm{hm}^{3} / \mathrm{año}$. Esto representa más del $10 \%$ del agua demandada por el regadío español, obtenido tanto a partir de la disminución de pérdidas en conducción y distribución, como por la limitación de los retornos de riegos.

Parece evidente que toda mejora y modernización de un regadío tradicional debe partir de un estudio de la situación en que se encuentra el uso del agua (Álvarez et al., 2005). Según Playán et al. (2000) el diagnóstico de los problemas concretos que afectan al manejo de los recursos hídricos permite proponer las soluciones precisas para conseguir una mayor eficiencia en el uso del agua por parte de los agricultores. En este sentido, distintos autores indican que el análisis de los condicionantes socio-económicos que inciden en la gestión del agua (Cancela et al., 2004) y la actitud de los agentes implicados, agricultores y Comunidades de Regantes, constituyen la base para alcanzar un desarrollo agrícola sostenible (Cuesta et al., 2005; Cancela et al, 2003).

El objetivo de este trabajo se concreta en la caracterización técnica y social del manejo del agua en un regadío tradicional, en concreto en la zona regable del Valle de Lemos (Lugo, España), definida en el PNR como zona susceptible de modernización. Esta caracterización debe incluir, además de los aspectos técnicos del riego, cuestiones relativas a la participación social. Nos interesa valorar, independientemente de manejo del agua, las características que definen los agentes sociales implicados y evaluar la incidencia social del regadío. Todo ello debe permitirnos determinar y valorar los aspectos que se deben considerar en la modernización de un regadío tradicional.

\section{Descripción del área de estudio}

La zona regable del Valle de Lemos (Lugo, España) está situada al sur de la provincia de Lugo, en la Comunidad Autónoma de Galicia (Figura 1). Esta zona, declarada en 1966 , contemplaba la puesta en regadío de unas 5.300 ha a partir de aguas fluyentes del río Cabe y aguas reguladas procedentes del río Mao (Cuesta et al, 2004a). La red de distribución principal está formada por tres canales principales con una longitud total de $78,5 \mathrm{~km}$. y con caudales que varían desde los $5,5 \mathrm{~m}^{3} / \mathrm{s}$ en cabecera hasta los $0,2 \mathrm{~m}^{3} / \mathrm{s}$ en su desagüe en el río Cabe (Figura 2). Asociadas a estos canales existe una red de acequias de diferente capacidad y distinto estado de conservación con $147.000 \mathrm{~m}$ aproximadamente (Cuesta et al, 2004b).

La zona se caracteriza por un clima que en su parte norte comienza siendo Mediterráneo templado y va transformándose hacia el sur en Templado frío. La temperatura media anual calculada para el periodo de referencia $1967 / 00$ es de $13,8^{\circ} \mathrm{C}$ con un valor máximo de $21,8^{\circ} \mathrm{C}$ en los meses de Julio y Agosto y un mínimo de $6,7^{\circ} \mathrm{C}$ en Enero. La precipitación efectiva anual media registrada este periodo es de $795,0 \mathrm{~mm}$. Es interesante destacar el carácter errático de las precipitaciones estivales (desde el 22 de junio hasta el 23 de septiembre) en la zona de estudio. Así, en el periodo 1967/2000 nos encontramos con un valor medio de $95,8 \mathrm{~mm}$, con un mínimo de $11,5 \mathrm{~mm}$, un máximo de $274,5 \mathrm{~mm}$ y una desviación estándar que alcanza el valor de 51,3 (Figura 3). La evapotranspiración de referencia anual (ETo), calculada mediante el método descrito por Hargreaves \& Samani (1985), para el periodo considerado es de $1.217,5 \mathrm{~mm}$.

En la zona de estudio nos encontramos básicamente con 3 tipos de suelos de interés para el regadío (Cuesta, 2001; Paz et al, 1996). Los suelos predominantes, tipo A, responden a texturas franco-arcillo-limosas y arcillo-limosas y no presentan ningún problema en cuanto a profundidades potenciales de enraizamiento con el $72,5 \%$ de la superficie regable. Los suelos con limitaciones moderadas, tipo B, para el cultivo de especies de enraizamiento profundo y texturas que van de franco-arcillo-limosas a franco-limosas
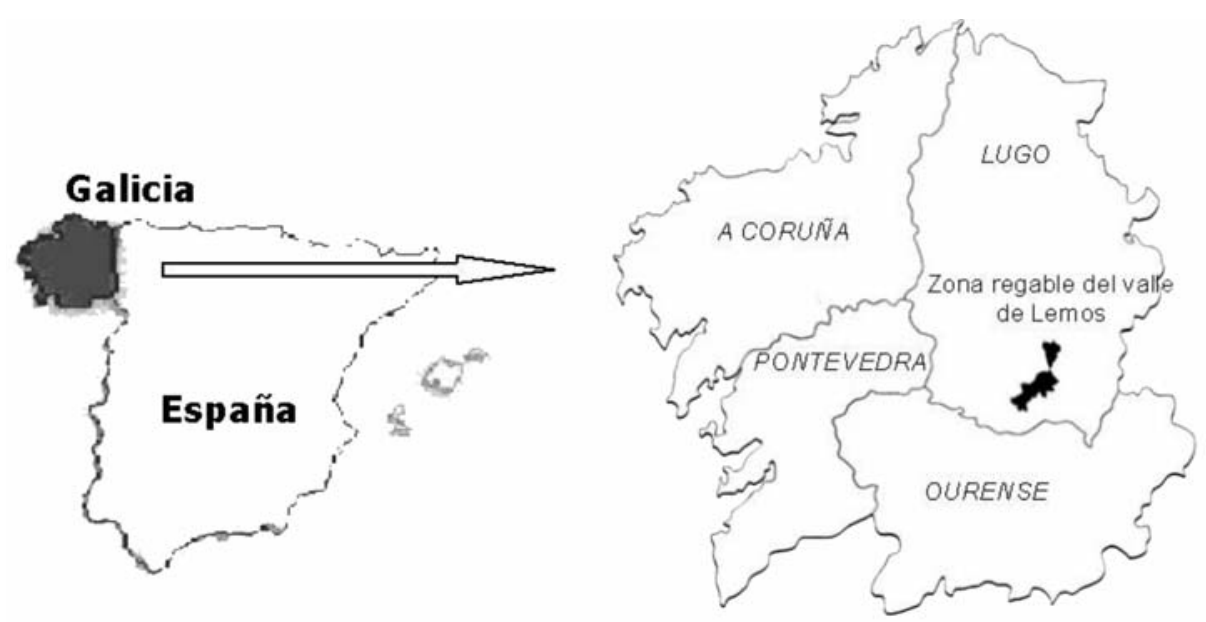

Figura 1.- Mapa de localización del área de estudio 


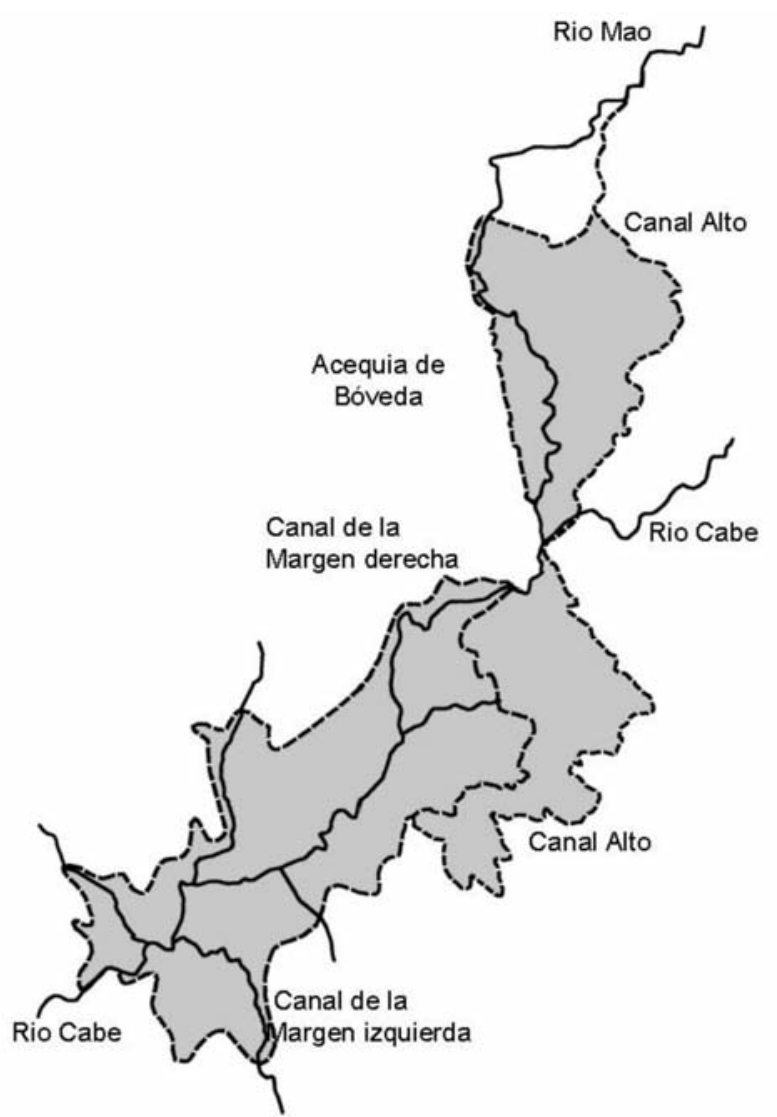

Figura 2.- Mapa de la red principal de distribución

representan el $12,1 \%$ del área estudiada. Distinguimos un último grupo de suelos con interés para el riego por superficie, tipo $\mathrm{C}$, con texturas franco-limosas, en zonas donde aparecen limitaciones claras para los cultivos de enraizamiento profundo que el $8,7 \%$ del conjunto de la superficie. El resto de los tipos de suelos existentes en la zona, que representan algo menos del 6,7\% del área de estudio, se excluyen de este trabajo al presentar serios problemas para el riego por gravedad. A partir de la metodología desarrollada por este equipo de investigación en otros trabajos (Cancela et al., 2006a), Neira et al. (2005) caracterizaron las propiedades de retención del agua a partir de 50 muestras tomadas en el conjunto de las unidades de suelos (Tabla 1).

El cultivo tradicional de la zona consiste en una rotación anual que combina la pradera artificial y el maíz forrajero, característica de una agricultura orientada a la ganadería. Destacan, por su importante presencia, los cultivos forrajeros de carácter anual, como el maíz y, ocasionalmente, el centeno, y algunas plantas forrajeras como pueden ser el nabo o la col (Cuesta et al., 2004b). En la actualidad, la mayoría de los riegos desarrollados corresponden a riego por gravedad en aquellas parcelas

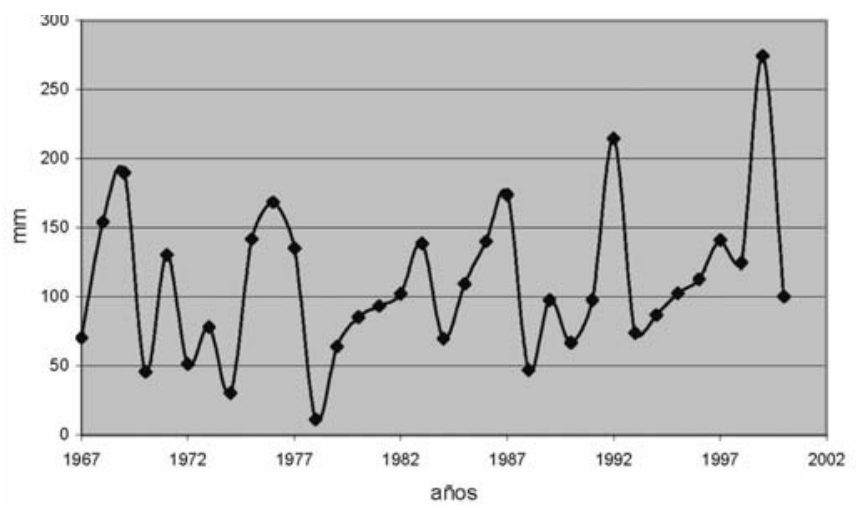

Figura 3.- Variación de la precipitación estival media (22 de junio a 23 de septiembre) en el período entre 1967 y 2000

que limitan con los canales, debido a la falta de una red de distribución en el perímetro de riego. Este último hecho obliga a que la conducción del agua a otras parcelas se realice a través de regueras en tierra con un consumo de agua excesivo, estimado en $12.500 \mathrm{~m}^{3} / \mathrm{ha}$ (Cancela et al., 2006b).

Desde el punto de vista demográfico, esta comarca cuenta con más de 30.000 habitantes con una importante y continua despoblación. En el conjunto de la zona regable tenemos 3.423 habitantes con 730 agricultores en el año 1999 (IGE, 2001).

\section{Metodología}

Para el estudio de la participación social y el manejo del riego se han realizado 119 encuestas sobre un total de 730 agricultores radicados en la zona regable durante el año 2000 (Cuesta et al., 2005). Además, con el fin de complementar la información obtenida de la encuesta, se realizaron evaluaciones de riego por superficie en nueve parcelas de ensayo repartidas entre las diferentes clases de suelos existentes (Neira et al., 2005).

\section{Evaluación de riego}

Para la realización de estas evaluaciones de riego es necesario determinar previamente las necesidades hídricas de los cultivos tradicionales de la zona. Los coeficientes de cultivo (Kc) utilizados se obtienen de otros trabajos realizados en Galicia (Cancela et al., 2006c, Cuesta, 2001). Además, se utilizaron los valores medios de la evapotranspiración del cultivo (ETc), la precipitación efectiva $(\mathrm{Pe})$ y las necesidades hídricas netas $(\mathrm{NHn})$ determinados en otros trabajos realizados en la misma zona de estudio (Neira et al., 2005; Cuesta, 2001). Las evaluaciones del riego por gravedad se realizaron siguiendo la metodología descrita por Pereira \& Trout (1999).

\begin{tabular}{ccccc}
\hline \multirow{2}{*}{ Tipo de suelos } & \multicolumn{2}{c}{ CC (\%) } & \multicolumn{2}{c}{ PMP (\%) } \\
\cline { 2 - 5 } & Media & Desviación & Media & Desviación \\
\hline A & 22.1 & 1.2 & 11.3 & 0.9 \\
\hline B & 20.3 & 0.7 & 8.7 & 0.7 \\
\hline C & 19.3 & 0.5 & 6.9 & 0.5 \\
\hline
\end{tabular}

Tabla 1.- Valores medios de capacidad de campo (CC) y punto de marchited permanente (PMP) para cada tipo de suelos en la zona de estudio (Neira et al., 2005) 
La forma y dimensiones de la parcela, así como la pendiente, se determinaron usando una estación total. Los caudales de entrada se midieron mediante lecturas de la velocidad del agua y de la sección mojada de los canales.

Se realizaron determinaciones de la infiltración en las parcelas evaluadas, mediante anillos infiltrómetros simples. A partir de las lecturas en los infiltrómetros se obtiene por regresión la ecuación de Kostiakov (Kostiakov, 1932). Para mejorar la significación de la ecuación, el valor de $\mathrm{k}$ de Kostiakov se ajusta para que la ecuación contenga al punto formado por la lámina de infiltración media y el tiempo de oportunidad medio (Cavero et al., 2000).

En las evaluaciones de riego se obtiene la longitud de parcela, el caudal unitario empleado, la SDe, la rugosidad del terreno, la pendiente de la parcela, los tiempos de avance del frente de agua y el tiempo de corte. El caudal unitario es el caudal por metro de anchura de la parcela. A partir de esta información, de los parámetros de las ecuaciones de Kostiakov ajustadas para cada tipo de suelo y de la $\mathrm{n}$ de Manning estimada se obtienen los índices de calidad del riego mediante el programa de simulación del riego por superficie SIRMOD (Walker, 1993). Los resultados obtenidos son los parámetros de eficiencia de aplicación, Ea, y uniformidad de distribución, UD, del riego simulado (Burt et al., 1997):

$$
\mathrm{Ea}=\frac{\text { volumen de agua retenido en la zona radicular }}{\text { volumen de agua aplicada }} \times 100
$$

UD $=\frac{\text { altura media de agua infiltrada en el } 25 \% \text { del área menos regada }}{\times 100}$ altura media de agua infiltrada en la parcela

Para las evaluaciones se seleccionaron nueve parcelas de diferentes dimensiones con cultivos de maíz y pradera en regadío, repartidas entre los suelos tipo $A, B$ y $C$.

\section{Encuesta a los regantes}

En la encuesta realizada por este este equipo de investigación se persigue recoger información relativa a las técnicas de riego empleadas e información relativa a la componente social del regadío (Cuesta et al., 2005). Para ello, se incluyen siete módulos diferenciados atendiendo a distintos aspectos del riego. El primer módulo contiene datos que permiten identificar y diferenciar la encuesta realizada. El segundo y tercer apartado proporcionan información sobre la estructura de la explotación y las características del titular que diferencian las explotaciones desde distintos supuestos sociales o económicos y comparan la idoneidad del conjunto de las explotaciones. El cuarto módulo recopila información general sobre la agricultura en secano y sus expectativas frente al regadío. La siguiente unidad se destina a las explotaciones que disponen de tierras en regadío y a cuestiones referentes al manejo del riego y al uso del agua. En el sexto apartado se agrupan una serie de preguntas relativas a la técnica de riego por superficie para cada cultivo. Finalmente, el último módulo empleado permite recoger impresiones y observaciones que, sin tener cabida en el formulario de la encuesta, se consideren de interés.

Con el objeto de obtener un nivel de significación del 90\%, se entrevistó a un número total de 119 agricultores sobre un total de 730 , lo que representa un porcentaje de error del $5,37 \%$.

\section{Resultados y discusión}

\section{Resultados de las evaluaciones de riego}

Las fincas donde se practicaron los ensayos presentan una pendiente longitudinal relativamente uniforme y superior al límite del 0,5 por ciento recomendado para el riego por escurrimiento (Playán et al., 2000). Así mismo, la mayoría de las parcelas de ensayo tienen una forma rectangular con anchuras excesivas. En efecto, mayoritariamente la relación entre el ancho y la longitud es superior al valor de 0,25 (Tabla 2).

La nivelación con rayo láser permite obtener parcelas aceptablemente niveladas, con valores de las desviaciones estándar de la elevación (SDe) en torno a $1 \mathrm{~cm}$ y con pendientes muy suaves para el riego por escurrimiento (Burt et al., 1997). Los resultados de la SDe obtenidos en las nivelaciones de las parcelas donde se evaluó el riego, son, en todos los casos, bastante negativos al ser superiores a los $4 \mathrm{~cm}$.

\begin{tabular}{cccccccccc}
\hline $\begin{array}{c}\text { Parcela } \\
\text { de }\end{array}$ & Cultivo & $\begin{array}{c}\text { Profundidad } \\
\text { del suelo } \\
\text { ensayo }\end{array}$ & $\begin{array}{c}\text { Pendiente } \\
(\mathbf{( \% )})\end{array}$ & $\begin{array}{c}\text { Superficie } \\
\left(\mathbf{m}^{\mathbf{2}} \mathbf{)}\right.\end{array}$ & $\begin{array}{c}\text { Longitud } \\
\mathbf{( m )}\end{array}$ & $\begin{array}{c}\text { Tiempo medio } \\
\text { de riego } \\
(\mathbf{m i n})\end{array}$ & $\begin{array}{c}\text { SDe } \\
\mathbf{( \mathbf { c m } )}\end{array}$ & $\mathbf{n}$ & $\begin{array}{c}\mathbf{Q} \\
(\mathbf{L} / \mathbf{s} \text { ha) }\end{array}$ \\
\hline 1 & Maiz & 70 & 1.03 & 11.484 & 175 & 380 & 5.7 & 0.03 & 63.93 \\
\hline 2 & Pradera & 25 & 1.54 & 8.060 & 130 & 190 & 9.6 & 0.15 & 72.42 \\
\hline 3 & Pradera & 30 & 0.54 & 6.580 & 140 & 250 & 5.8 & 0.21 & 99.12 \\
\hline 4 & Maiz & 80 & 2.32 & 6.750 & 150 & 495 & 4.6 & 0.06 & 79.23 \\
\hline 5 & Maiz & 60 & 0.94 & 15.540 & 210 & 720 & 7.8 & 0.07 & 56.31 \\
\hline 6 & Pradera & 30 & 0.78 & 14.744 & 195 & 205 & 6.2 & 0.12 & 21.57 \\
\hline 7 & Pradera & 35 & 1.25 & 5.920 & 160 & 240 & 8.9 & 0.13 & 82.72 \\
\hline 8 & Maiz & 90 & 1.23 & 8.845 & 145 & 645 & 7.9 & 0.04 & 44.23 \\
\hline 9 & Pradera & 30 & 0.85 & 8.160 & 170 & 280 & 10.6 & 0.15 & 70.11 \\
\hline
\end{tabular}

Tabla 2.- Parametros empleados en las evaluaciones: desviación standard de la nivelación (SDe), coeficiente de rugosidad de Manning ( $\mathrm{n}$ ) y caudal de riego ( $\mathrm{Q}$ ) 


\begin{tabular}{c|ccccc}
\hline $\begin{array}{c}\text { Parcela de } \\
\text { ensayo }\end{array}$ & $\begin{array}{c}\mathrm{Vt} \\
\left(\mathbf{m}^{3} / \mathbf{m}^{2}\right)\end{array}$ & $\begin{array}{c}\mathrm{Vi} \\
\left(\mathbf{m}^{3} / \mathbf{m}^{2}\right)\end{array}$ & $\begin{array}{c}\text { Vs } \\
\left(\mathbf{m}^{\mathbf{3}}\right)\end{array}$ & $\begin{array}{c}\text { Ea } \\
(\%)\end{array}$ & $\begin{array}{c}\text { UD } \\
(\%)\end{array}$ \\
\hline 1 & 0.146 & 0.139 & 86 & 48.3 & 74.4 \\
\hline 2 & 0.082 & 0.049 & 267 & 36.3 & 96.7 \\
\hline 3 & 0.149 & 0.066 & 541 & 20.2 & 98.8 \\
\hline 4 & 0.235 & 0.152 & 560 & 25.5 & 92.2 \\
\hline 5 & 0.243 & 0.219 & 366 & 24.7 & 80.9 \\
\hline 6 & 0.026 & 0.020 & 81 & 79.1 & 91.6 \\
\hline 7 & 0.119 & 0.073 & 270 & 21.0 & 94.4 \\
\hline 8 & 0.171 & 0.163 & 64 & 40.9 & 75.0 \\
\hline 9 & 0.118 & 0.092 & 205 & 21.2 & 90.1 \\
\hline
\end{tabular}

Tabla 3.- Resultados de las evaluaciones de riego en las parcelas de ensayo: Volumen total aplicado durante el riego $(\mathrm{Vt})$, volumen infiltrado $(\mathrm{Vi})$, volumen de escorrentía (Vs), eficiencia de aplicación (Ea) y uniformidad de distribución (UD)

La simulación del riego, realizada con los datos obtenidos en las evaluaciones, nos proporciona el valor del volumen total de riego, el volumen infiltrado y el volumen de escorrentía superficial. A partir de esos valores podemos obtener la eficiencia de aplicación, Ea y la uniformidad de distribución, UD para cada evaluación realizada (Tabla 3).

Los valores de Ea obtenidos en casi todos los ensayos pueden considerarse bajos e incluso muy bajos (Cavero et al., 2001; Burt et al., 1997). El valor medio resultante alcanza el $35,2 \%$, con un mínimo de $20,2 \%$ y un máximo de $79,1 \%$. El valor máximo de eficiencia, $79,1 \%$, corresponde a la parcela número 6 donde el agua aplicada sólo cubre el $69,2 \%$ del volumen de déficit hídrico en la zona radicular.

Los resultados correspondientes a los ensayos realizados en suelos del grupo A presentan valores de eficiencia muy bajos, excepto las evaluaciones realizadas en la parcela 8 . En lo que respecta a los valores de Ea correspondientes a suelos tipo $B$ y $C$ parece que no puede obtenerse ninguna conclusión dada la disparidad de los resultados y el número de ensayos realizados.

Los valores de UD obtenidos pueden considerarse como normales a excepción de los correspondientes a las fincas de ensayo 1 y 8 que resultan inferiores al $80 \%$ (Pereira \& Trout, 1999). Este resultado parece lógico dado el alto volumen de agua total aplicado generalmente en los diferentes riegos.

\section{Características sociales de las explotaciones}

Las explotaciones elegidas representan, en su conjunto, 998 parcelas con una superficie total de 340,48ha. La superficie media de la parcela es de $3.412 \mathrm{~m}^{2}$ y cada explotación está formada por una media de 8,4 parcelas.

A pesar de la escasa superficie media de las parcelas y del elevado número de parcelas por explotación, únicamente el $13,3 \%$ de las explotaciones encuestadas se ha visto afectada por procesos de Concentración Parcelaria. Al preguntar a los titulares no afectados por la Concentración Parcelaria, el $80,8 \%$ manifiesta su interés por la reestructuración de la propiedad en sus explotaciones. Esta actuación pública es necesaria en esta zona regable aunque las explotaciones y las fincas concentradas seguirían teniendo un tamaño demasiado pequeño para el sustento familiar sin una intensificación suficiente de cultivos (Crecente et al., 2002).

En lo concerniente a la edad de los agricultores, un $66,6 \%$ de los propietarios se caracterizan por tener menos de 55 años, de lo que se deduce indirectamente la existencia de una garantía de continuidad a medio plazo (Figura 4). Estos porcentajes coinciden con la distribución demográfica media registrada en Galicia, donde se experimenta una importante y continua despoblación acentuada en las zonas interiores. Al preguntarles directamente a los mayores de 55 años $(33,3 \%)$ acerca de la posibilidad de continuidad de la explotación una vez que ellos abandonen la actividad agraria, sólo el $30 \%$ responde positivamente y un $20 \%$ expresa su incertidumbre ante el futuro.

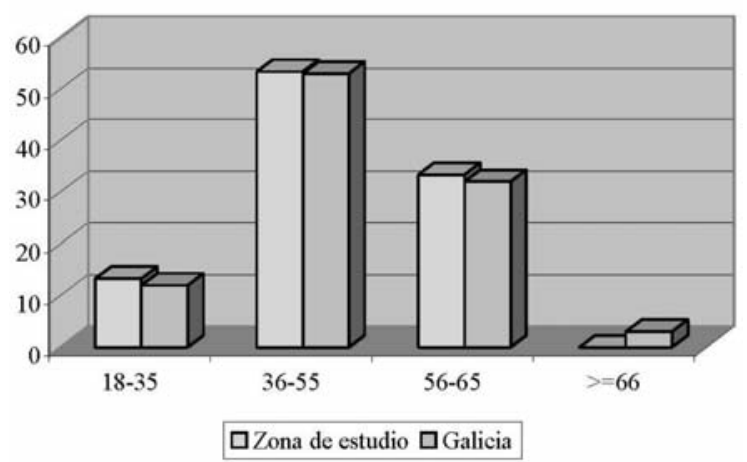

Figura 4.- Distribución por rangos de edad de los titulares de las explotaciones

Respecto a la distribución por sexo de los titulares de explotación, los porcentajes obtenidos son muy similares mientras que en la comunidad gallega, el porcentaje de hombres es inferior al de mujeres (Figura 5). En este sentido, mientras que en Galicia lo normal es que la mayoría los propietarios sean agricultores a tiempo completo (91,5\%), aquí nos encontramos únicamente con un $72,3 \%$. Esto explica que sólo el $33,4 \%$ de los casos deban la totalidad de sus ingresos a la explotación.

Las unidades familiares características de la zona tienen un tamaño medio de cinco personas, destacando que el $69,7 \%$ de las familias son de 3 y 5 personas (Figura 6). Este dato tiene gran significación en lo que se refiere al trabajo en la explotación. Así en un $46,7 \%$ de los casos el cónyuge trabaja en la propia explotación, siendo la mano de obra asalariada muy escasa $(13,3 \%)$. 


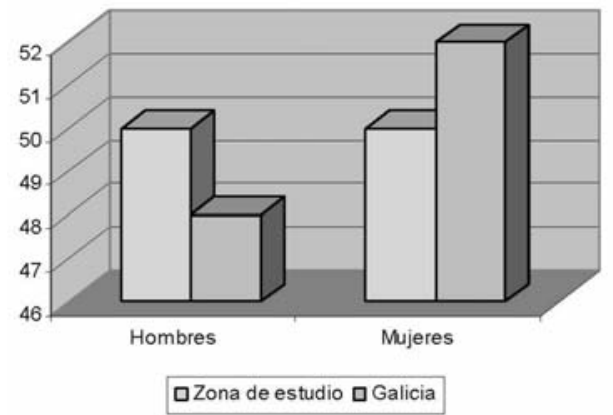

Figura 5.- Distribución por sexo de los titulares de las explotaciones

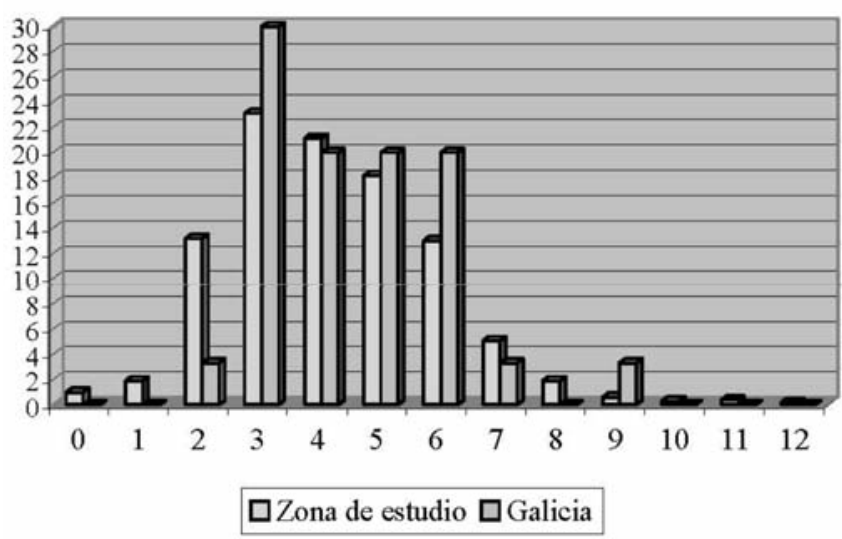

Figura 6.- Número de personas que forma la unidad familiar

En general el nivel de formación de los agricultores es bajo aunque superior a la media gallega (Tabla 4). El 76,5\% de los agricultores sólo posee estudios básicos.

\begin{tabular}{ccccc}
\cline { 2 - 5 } & \multicolumn{2}{c}{ Zona estudio } & \multicolumn{2}{c}{ Galicia } \\
\cline { 2 - 5 } & Frecuencia & $\%$ válido & Frecuencia & $\%$ válido \\
\hline Sin estudios & 7 & 5.9 & 717 & 20,1 \\
\hline Básica & 91 & 76,5 & 2477 & 69,5 \\
\hline Media & 20 & 16,8 & 338 & 9,5 \\
\hline Superior & 1 & 0,8 & 31 & 0,9 \\
\hline Total respuestas & 163 & 100,0 & 3563 & 100,0 \\
\hline
\end{tabular}

Tabla 4.- Nivel de formación del titular (estudios generales)

Desde el punto de vista económico se observa que la gran mayoría de los encuestados se concentran en los dos intervalos más bajos de ingresos, menos de $1800 €$ por mes y explotación, representando un $86,7 \%$ sobre el total (Figura 7).

\section{Conclusiones}

Los valores de eficiencia de aplicación (Ea) obtenidos resultan bajos o muy bajos, con un valor medio del $35,2 \%$. Los resultados correspondientes a los ensayos realizados en los suelos con más aptitud para el riego presentan valores de eficiencia muy bajos. En cuanto a los valores de

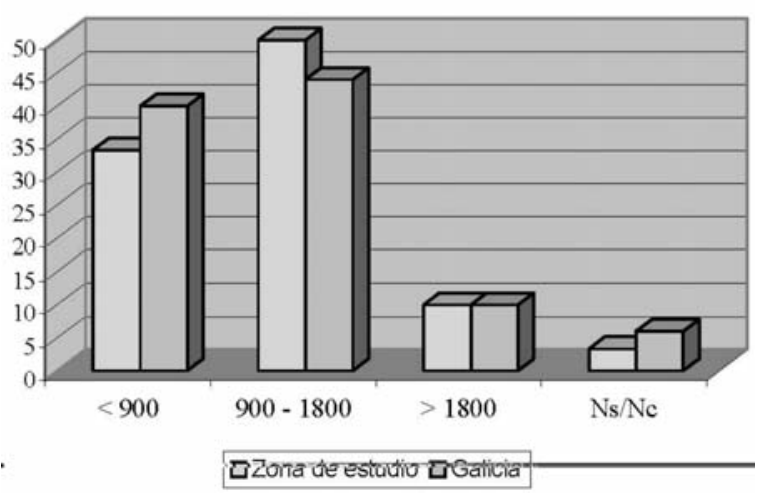

Figura 7.- Nivel de ingresos netos de la unidad familiar

Ea correspondientes al resto de los tipos de suelo identificados es conveniente realizar un mayor número de pruebas para establecer conclusiones fiables. En lo que respecta a los valores de uniformidad de distribución (UD), estos se consideran normales con alguna excepción.

Con una relación entre el ancho y la longitud de los tablares entre el 0,1 y el 0,2 se puede multiplicar por dos o por tres el caudal unitario, lo que permitiría conseguir un frente de avance del agua uniforme. El incremento de caudal mejora los resultados primitivos de Ea y del tiempo de corte y, sobre todo, reducir significativamente el volumen de agua destinado al riego. Con el incremento de caudal los valores de UD se mantienen aunque se pueden mejorar con una nivelación de las parcelas. Para actuar sobre las pendientes, ligeramente altas, hay que valorar el coste y el impacto ambiental.

A pesar del interés de la administración por la mejora y modernización del regadío en el Valle de Lemos, plasmado en el Plan Nacional de Regadíos y en la declaración de interés general de la mejora del sistema de riego, existen dos aspectos que limitan o condicionan esta actuación. En primer lugar destaca el retroceso del riego en esta zona. El otro elemento limitante se deriva de la estructura de la propiedad y de la escasa dimensión de las explotaciones.

Un problema existente en este regadío tradicional es la alta parcelación de las fincas que obliga al regante a realizar frecuentes desplazamientos, según el número de fincas y su dispersión espacial. El elevado número de parcelas, la forma irregular de las mismas y su reducida superficie, complican la gestión del agua en el conjunto de la zona regable y crean la necesidad de sistemas complejos de distribución, extensas acequias de drenaje y densas redes de caminos. La Concentración Parcelaria parece totalmente necesaria en esta zona regable aunque las fincas concentradas seguirían teniendo un tamaño pequeño. Por otro lado, la superficie media por explotación en esta zona no alcanza las 3 ha, por lo es necesaria la concentración de explotaciones.

Parece claro que cualquier inversión pública encaminada a la mejora y modernización del regadío en el valle de Lemos ha de tener en consideración estos dos aspectos limitantes. Así mismo, esta actuación de la administración debe aprovecharse para introducir los elementos de la gestión sostenible, tal y como se persigue en la DMA. 


\section{Bibliografía}

Aguilera, F. (1994). Agua, economía y medio ambiente: interdependencias físicas y necesidad de nuevos conceptos. Revista de Estudios Agrosociales. 167: 113-130.

Álvarez, C.J., Cancela, J.J. \& Fandiño, M. (2005). Characterization of irrigation holdings in the Terra Cha region of Spain: a first step towards a water management model. Water Resources Management 19, 1: 23-36.

Arrojo, P. (2003). Spanish National Hydrological Plan: Reasons for its failure and arguments for the future. Water International. 28: 295-302.

Beceiro, M.S. (2003). Legal considerations of the 2001 National Hydrological Plan. Water International. 28, 3: 303312.

Burt, C.M., Clemmens, A.J., Strelkoff, T.S., Solomon, K.H., Bliesner, R.D., Ardí, L.A., Howell, T.A. \& Eisenhauer, D.E. (1997). Irrigation performance measures: Efficiency and uniformity. Journal Irrigation and Drainage Engineering ASCE. 123, 6: 423-442.

Cancela J.J., Dafonte, D., Martínez, E.M., Cuesta, T.S. \& Neira, X.X. (2006a). Assessment of a Water Activity Meter for Rapid Measurements of Soil Water Potential. Biosystem Engineering. 94, 2: 285-295.

Cancela J.J., Cuesta, T.S., Fandiño, M., Martínez, E.M. \& Neira, X.X. (2006b). Caracterización de las zonas regables de la Provincia de Lugo. Riegos y Drenajes XXI. 148: 50-56.

Cancela J.J., Cuesta, T.S., Neira, X.X. \& Pereira, L.S. (2006c). Modelling for improved irrigation water management in a temperate region of Northern Spain. Biosystem Engineering. 94, 1: 151-163.

Cancela, J.J., Neira, X.X., Cuesta, T.S., Álvarez, C.J. \& Crecente, R. (2004). Socio-Economic Evaluation of the Terra Cha Irrigators Community by using a Geographic Information System-Spain. Agricultural Engineering International: the CIGR Journal of Scientific Research and Development. Volume VI July 2004.

Cancela, J.J., Neira, X.X., Cuesta, T.S., Álvarez, C.J. \& Crecente, R. (2003). Evaluation by Scientific Inquiry and Geographic Information System of Terra Chá Irrigators Community. VI Inter.-Regional Conference on Environment Water, Albacete, Spain.

Cavero, J., Playán, E., Zapata, N. \& Faci, J.M. (2001). Simulation of maize Grain Yield Variability within a SurfaceIrrigated Field. Agronomy Journal. 93: 773-782.

Crecente, R., Álvarez, C. \& Fra, U. (2002). Economics, social and envirenmental impact of land consolidation in Galicia. Land Use Policy. 19: 135-147.

Cuesta, T.S. (2001). Gestión y uso del agua en la zona regable del Valle de Lemos. Servicio de Publicaciones e Intercambio Científico, Universidad de Santiago de Compostela, Santiago de Compostela.
Cuesta, T.S., Cancela, J.J., J. Dafonte, M. Valcárcel \& Neira, X.X. (2005). Social aspects influencing water management in the Lemos Valley Irrigation District, Spain. Irrigation and Drainage. 54, 2: 125-133.

Cuesta, T.S., Neira, X.X. \& Cancela, J.J. (2004a). Las grandes zonas regables de Galicia. Agricultura Revista Agropecuaria. 865: 650-654.

Cuesta, T.S., X.X. Neira \& Cancela, J.J. (2004b). Gestión del agua en la zona regable del Valle de Lemos (Lugo). Riegos y Drenajes XXI. 136: 64-71.

De Miguel, J.C., Pérez, T., Rodríguez, X.A. (2003). Tendencias productivas en las explotaciones de leche gallegas. Revista Galega de Economía. 12, 1: 1-18.

Del Moral, L., Van der Werff, P., Bakker, K. \& Handmer, J. (2003). Global trends and water policy in Spain. Water International. 28, 3: 358-366.

Hargreaves, G.L. \& Samani, Z.A. (1985). Reference crop evapotranspiration from temperature. Applied Engineering in Agriculture. 1, 2: 96-99.

IGE (2001). Padrón Municipal de habitantes 1996, 1998 y 1999. Instituto Galego de Estatística, Santiago de Compostela.

Kostiakov, A.N. (1932). On the dynamics of the coefficient of water-percolation in soils and on the necessity for studying it from a dynamic point of view for purposes of amelioration. International Congress Soil Sciences, Paris, France.

Loucks, D.P. (2000). Sustainable Water Resources Management. Water International. 25: 3-10.

MAPA (2001). Plan Nacional de Regadíos. Horizonte 2008, Dirección General de Desarrollo Rural, Subsecretaria, Ministerio de Agricultura, Pesca y Alimentación, Madrid.

Martín de Santa Olalla, F., Brasa, A., Fabeiro, C., Fernández, D. \& López, H. (1999). Improvement of irrigation management towards the sustainable use of groundwater in Castilla-La Mancha, Spain. Agricultural Water Management. 40: 195-205.

MIMAM (1998). Libro Blanco del Agua, Documento de Síntesis. Ministerio de Medio Ambiente, Madrid.

Neira, X.X., Álvarez, C.J., Cuesta, T.S. \& Cancela, J.J. (2005). Evaluation of water use in traditional irrigation: an application to the Lemos Valley Irrigation District, northwest of Spain. Agricultural Water Management. 75, 2: 137-151.

Ortega, J.F., de Juan, J.A., Chillarón, M., Tarjuelo, J.M. \& Artigao, A. (2000). El uso del agua con fines de interés social: rehabilitación de regadíos en zonas desfavorecidas y su integración en programas de Desarrollo Rural. XVIII Congreso Nacional de Riegos, Huelva, 143-145.

Paz, A., Neira X.X. \& Castelao, A. (1996). Soil water regime under pasture in the humid zone of Spain: Validation of an empirical model and prediction of irrigation requirements. Agricultural Water Management. 29, 2: 147-161. 
Pereira, L.S. \& Trout, T.J. (1999). Irrigation Methods. Irrigation and Drainage. En: Handbook of Agricultural

Engineering. Ed. CIGR-The International Commission of

Playán, E., Slatni, A., Castillo, R. \& Faci, J.M. (2000). A case

Agricultural Engineering. ASCE, 297-379.

study for irrigation modernisation II. Scenario analysis.

Agricultural 335-354. 